\title{
Role of c-Fos in orthodontic tooth movement: an in vivo study using transgenic mice
}

\author{
Maximilian G. Decker ${ }^{1} \cdot$ Cita Nottmeier ${ }^{1} \cdot$ Julia Luther $^{2} \cdot$ Anke Baranowsky $^{2} \cdot$ Bärbel Kahl-Nieke $^{1} \cdot$ Michael Amling $^{2}$. \\ Thorsten Schinke ${ }^{2} \cdot$ Jean-Pierre David ${ }^{2} \cdot$ Till Koehne $^{1}$ (D)
}

Received: 30 March 2020 / Accepted: 5 August 2020 / Published online: 15 August 2020

(C) The Author(s) 2020

\begin{abstract}
Objectives The transcription factor $c$-Fos controls the differentiation of osteoclasts and is expressed in periodontal ligament cells after mechanical stimulation in vitro. However, it is unclear how c-Fos regulates orthodontic tooth movement (OTM) in vivo. The aim of this study was therefore to analyse OTM in transgenic mice with overexpression of $c-F o s$.

Materials and methods We employed $c-F o s$ transgenic mice ( $c$-Fos tg) and wild-type littermates (WT) in a model of OTM induced by Nitinol tension springs that were bonded between the left first maxillary molars and the upper incisors. The unstimulated contralateral side served as an internal control. Mice were analysed by contact radiography, micro-computed tomography, decalcified histology and histochemistry.

Results Our analysis of the unstimulated side revealed that alveolar bone and root morphology were similar between c-Fos tg and control mice. However, we observed more osteoclasts in the alveolar bone of $c$-Fos tg mice as tartrate-resistant acid phosphatase (TRAP)-positive cells were increased by $40 \%$. After 12 days of OTM, $c$-Fos tg mice exhibited $62 \%$ increased tooth movement as compared with WT mice. Despite the faster tooth movement, $c-F o s$ tg and WT mice displayed the same amount of root resorption. Importantly, we did not observe orthodontically induced tissue necrosis (i.e. hyalinization) in $c$-Fos tg mice, while this was a common finding in WT mice.

Conclusion Overexpression of c-Fos accelerates tooth movement without causing more root resorption.

Clinical relevance Accelerated tooth movement must not result in more root resorption as higher tissue turnover may decrease the amount of mechanically induced tissue necrosis.
\end{abstract}

Keywords Orthodontic tooth movement $\cdot \mathrm{c}$-Fos $\cdot$ Mechanical stimulation $\cdot$ Bone remodelling $\cdot$ Root resorption

\section{Introduction}

Orthodontic tooth movement (OTM) is a prime example of mechanically induced bone remodelling. The transduction of mechanical stimuli into differentiation and activity of bonebuilding osteoblasts and bone-degrading osteoclasts is

Electronic supplementary material The online version of this article (https://doi.org/10.1007/s00784-020-03503-1) contains supplementary material, which is available to authorized users.

Till Koehne

tkoehne@uke.de

1 Department of Orthodontics, University Medical Center Hamburg-Eppendorf, Martinistr. 52, 20246 Hamburg, Germany

2 Institute of Osteology and Biomechanics, University Medical Center Hamburg-Eppendorf, Hamburg, Germany regulated by a variety of genetic and epigenetic factors [1]. This explains why the biological response to mechanical forces can significantly vary from one patient to another [2]. In fact, significant differences have been observed among patients with regard to the velocity of OTM [3, 4] or the occurrence of adverse effects such as root resorption [5]. Since these differences require a patient-specific management in orthodontic care, there is a clear need towards a deeper understanding of the biological principles of tooth movement.

Recently, genetically modified mice were used to study the genetic basis of tooth movement [6-11]. Whereas larger animal models are easier to handle, only mice offer the possibility to analyse the role of single genes during OTM in vivo. Mouse models are therefore a valuable approach to decrease the gap that exists between our knowledge from in vitro studies and the small list of genes that are actually known to regulate OTM in vivo. 
Of particular interest in this regard is $c-F o s$, a member of the AP-1 transcription factor family. $C$-fos is activated in osteoclast precursors and is required for osteoclast differentiation [12]. Deletion of $c-F o s$ in mice leads to osteopetrosis, a phenotype characterized by abnormally high bone mass due to disturbed bone resorption [13, 14]. Conversely, overexpression of $c$-Fos in mice leads to the development of chondrogenic tumours [15]. These tumours are also evident in the occipital bones of the skull, which ossify through endochondral ossification. However, it is unclear whether $c-F o s$ overexpression also affects the jaw bones, which ossify through intramembranous ossification.

Interestingly, $c-F o s$ was also identified as a key mechanosensor in early gene transcription after mechanical loading [16]. In fact, numerous in vitro studies have demonstrated that mechanical forces result in an upregulation of $c$ Fos in various cell types including osteocytes $[17,18]$, osteoblasts [19, 20], and periodontal cells [21-23]. In particular, compression or extension of periodontal ligament cells leads to an induction of $C-F O S$ on the RNA and protein level [21-23]. Although these studies clearly suggest that c-Fos plays a key role in OTM, it remains to be established whether and how c-Fos controls OTM in vivo.

The aim of this study was therefore to analyse the role of $c$ Fos in OTM by using mice with overexpression of $c-F o s$ ( $c$ Fos tg) and control littermates in a mouse model of OTM.

\section{Materials and methods}

\section{Mice}

C-fos transgenic mice (c-Fos tg) were maintained on a $\mathrm{C} 57 \mathrm{BL} / 6 \mathrm{~J}$ background and fed a soft rodent diet. The transgenic mice overexpress the $c$-Fos gene under the control of the glucocorticoid- and heavy metal-inducible human metallothionein promoter, which is ubiquitously expressed [24-27]. Wild-type littermates (WT) served as controls and only females were used. The orthodontic appliance was applied to the mice while they were under anaesthesia and at 10 weeks of age (Fig. 1a). After 12 days of OTM, all mice were euthanized by $\mathrm{CO}_{2}$ inhalation. Animal treatment procedures were approved by the commission for animal welfare (Behörde für Gesundheit und Verbraucherschutz der Hansestadt Hamburg, Nr. 121/16).

\section{Orthodontic appliance}

All mice were anaesthetized by intraperitoneal injection with $10 \mathrm{ml} / \mathrm{kg}$ anaesthetic mixture $(40 \mathrm{mg} / \mathrm{kg}$ bw ketamine-S, xylazine $2 \% 16 \mathrm{mg} / \mathrm{kg}$ bw, heparin $40,000 \mathrm{IE} / \mathrm{kg}$ bw in $0.9 \% \mathrm{NaCl}$ ). The mice were carefully fixed on a modified miniature lathe (Unimat 3, Emco, Wiener Neudorf, Austria).
The cheeks were gently spread with a specially designed carriage holder and the upper jaw was carefully fixed to the operating table using a loop made of suture material (Vicryl, Ethicon Inc, New Jersey, USA). The incisors of the mandible were then inserted into the eyelet of an orthodontic rubber chain (Elasto-Force, Dentaurum, Ispringen, Germany) and fixed without tension. The tooth surfaces of the first left molar and both maxillary incisors were etched with $37 \%$ phosphoric acid gel (HS-etchgel 37\%, Henry Schein Dental, Langen, Germany) (Fig. 1a and b). After $30 \mathrm{~s}$, the gel was removed with microbrushes and the tooth surfaces were cleaned and dried successively with ethanol and specially prepared paper tips. Bonding (Scotchbond, $3 \mathrm{~m}$ Espe, Neuss, Germany) was applied and polymerized (Fig. 1b). The spring was placed with its distal end on the first molar and fixed with a lightcuring composite (Estelite Flow Quick, Tokuyama Dental Corp., Tokyo, Japan). To activate the spring, the operating table was moved parallel to the planned force direction until the desired force of 35 centinewton was reached (tension gauge, Dentaurum, Ispringen, Germany) [9]. The mesial part of the spring was then fixed to both maxillary incisors with a light-curing composite (Estelite Flow Quick, Tokuyama Dental Corp., Tokyo, Japan) (Fig. 1b and c). After surgery, mice were transferred to a heat mat and monitored until they fully recovered from anaesthesia.

\section{Micro-CT and histological analysis}

After 12 days of OTM, all mice were euthanized and fixed in $4 \%$ PB-buffered formaldehyde for $24 \mathrm{~h}$. The skulls were removed and analysed by contact radiography using a Faxitron X-ray cabinet (Faxitron X-ray Corp., Wheeling, IL, USA). Xray microtomography $(\mu \mathrm{CT})$ of the skulls was performed with a $\mu$ CT 40 scanner (Scanco Medical, Bassersdorf, Switzerland). Images were constructed at a spatial resolution of $15 \mu \mathrm{m}$. Exposed surfaces of the roots were highlighted on $\mu \mathrm{CT}$ images using Photoshop (Photoshop Cs 6, Adobe Systems Inc., USA). Orthodontic tooth movement and alveolar bone loss were evaluated on $\mu$-CT images using ImageJ 1.52 (National Institutes of Health, Bethesda, MD, USA). Orthodontic tooth movement was defined as the shortest distance between the crowns of the maxillary first and second molars measured on $\mu$-CT cross-sections. Alveolar bone loss was defined as the area of the exposed root surface measured on $\mu$-CT three-dimensional reconstructions. For histology, skulls were decalcified for 14 days in Usedecalc (MEDITE Medical GmbH, Burgdorf, Germany), dehydrated in ascending alcohol concentrations, and embedded in paraffin. Fourmicrometre-thick sections were cut on a microtome (Supercut 2050, Reichert-Jung, Leica Microsystems GmbH, Wetzlar, Germany). Slides were deparaffinized in xylene and stained with toluidine blue (1\%, pH 4.5) for $30 \mathrm{~min}$. For TRAP staining, slides were deparaffinized and stained with TRAP for 
a
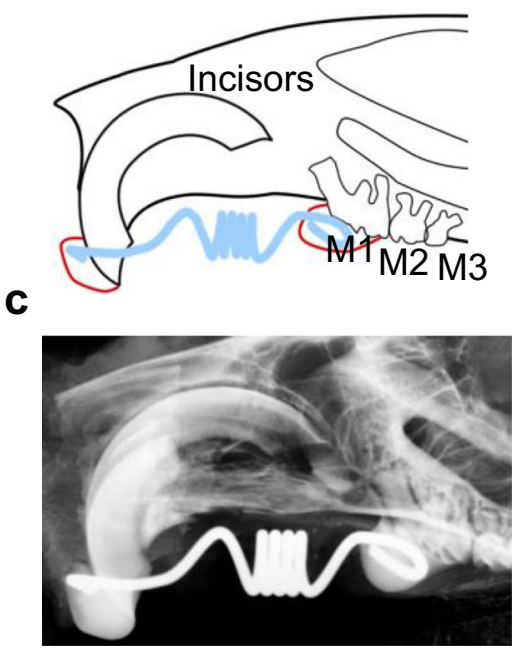

Fig. 1 Overexpression of $c$-Fos accelerates orthodontic tooth movement in mice. a Schematic drawing of the OTM model. The Nitinol spring (blue) was bonded to the incisors and to the left first molar (M1) with a light-curing composite (red circles). b Photographs of the bonding procedure. The first molar (red arrow) was dried (upper left panel) and etched (upper right panel)

b
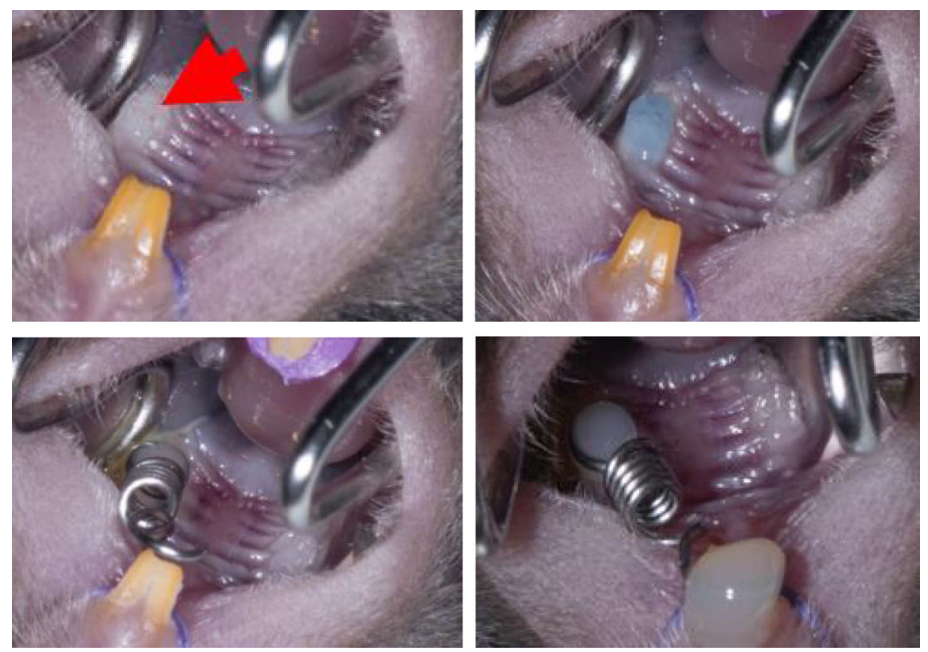

before the mesial end of the spring was bonded (lower left panel). After activation of the spring with a force of 35 centinewton, the distal end of the spring was bonded to the incisors (lower right panel). c Contact radiography showing the activated nitinol spring
$120 \mathrm{~min}$ at $37^{\circ} \mathrm{C}(50 \mathrm{ml}$ TRAP solution: $5 \mathrm{mg}$ Naphtol-ASMX phosphate dissolved in $500 \mu$ l dimethyl formamide, $30 \mathrm{mg}$ Fast Red Violet; $40 \mathrm{mM}$ sodium acetate and $10 \mathrm{mM}$ sodium tartrate as buffer). Histomorphometric quantification was performed using the Osteo-Measure histomorphometry system (Osteometrics, Atlanta, GA, USA).

\section{Statistics}

The statistical analysis of the data as well as their graphic representation was carried out with the software GraphPad PRISM (GraphPad Software, San Diego, USA). A twosided $t$ test was used for statistical testing of independent samples. ANOVA with Bonferroni post hoc test was used for multi-group comparisons. $P$ values below 0.05 were considered statistically significant. All graphs show mean values with standard deviations.

\section{Results}

We first examined the teeth and alveolar bone of $c$-Fos tg and WT mice without orthodontic tooth movement (OTM-) using $\mu-\mathrm{CT}$ imaging (Fig. 2). We observed that tooth morphology, alveolar bone, and palatal bone of $c-F o s$ tg were similar to those of controls (Fig. 2a). In fact, quantification of alveolar bone loss (ABL) and palatal thickness revealed no differences between $c$-Fos tg and WT mice (Fig. 2b and c). We next determined the effect of orthodontic tooth movement (OTM+) in c-Fos and control mice (Fig. 2d, lower panels). We observed that OTM resulted in a separation of the 1st and 2nd molar crowns, which was significantly wider in $c-F o s$ tg mice as compared with those of controls. In fact, quantification of the shortest distance between the 1st and 2nd molars revealed $62 \%$ increased tooth movement in $c$-Fos tg mice (Fig. 2e). Taken together, these first analyses suggest that $c$ Fos overexpression increases OTM and that this acceleration cannot be explained by differences in alveolar bone architecture between $c$-Fos tg and WT mice.

We therefore next performed a detailed histological analysis of OTM in c-Fos tg and WT mice (Fig. 3). In this regard, it is important to consider that OTM causes zones of compression and tension in the periodontal ligament, which have to be analysed separately (Fig. 3a). Before we looked in more detail in the histological appearance caused by OTM, we analysed toluidine-blue stained histological sections of teeth without OTM from $c-F o s$ tg and WT mice (Fig. 3b). The morphology and structure of bone and teeth appeared histologically normal in $c-F o s \operatorname{tg}$ mice. We again observed that OTM caused a larger intercoronal gap in c-Fos tg mice as compared with controls (Fig. 3c). This was associated with food impaction causing an inflammatory epithelial thickening of the gingival papilla (Suppl. Fig. 1). We next focused on the distal root of the 1st molar, where the periodontal ligament (PDL) on the distal side is subjected to tension, whereas the PDL on the mesial side is subjected to compression (Fig. 3d). Without OTM, we found only some active bone cells in the PDL of $c$-Fos tg and control mice (Fig. 3e). The bone surface was mainly covered by nonactive bone-lining cells. In contrast, after OTM numerous bone-forming osteoblasts were evident in the tensile zone of both $c-F o s$ tg and control mice (Fig. 3f, upper panels). The cubic shape and arrangement of the osteoblasts clearly 
Fig. 2 Micro-CT analysis of alveolar bone, tooth structure and OTM. a Micro-CT scanning of maxillary molars from 12-weekold wild-type (WT) and $c$-Fos transgenic (c-Fos tg) mice. Alveolar bone loss (highlighted in red) was measured on 3D reconstructions of teeth that were not subjected to OTM (upper panels). Palatal thickness was measured on cross-sections of the palate (lower panels). Scale bars $=3$ $\mathrm{mm}$. b, c Quantification of the alveolar bone loss (b) and palatal thickness (c) of 12-week-old WT and $c$-Fos tg mice. $n \geq 3$. $* P<$ 0.05 , versus WT. d Crosssections based on micro-CT scans of untreated (OTM-) and treated $(\mathrm{OTM}+)$ maxillary molars of wild-type (WT) and c-Fos transgenic mice (c-Fos tg). The mechanical loading created a gap between the first and second molar (red arrows). Scale bars $=1$ $\mathrm{mm}$. e Quantification of the smallest distance between the first and second molar as a surrogate measurement for OTM in 12week-old wild-type (WT) and $c$ Fos tg mice. $n=4$. $* P<0.05$, versus wild-type a
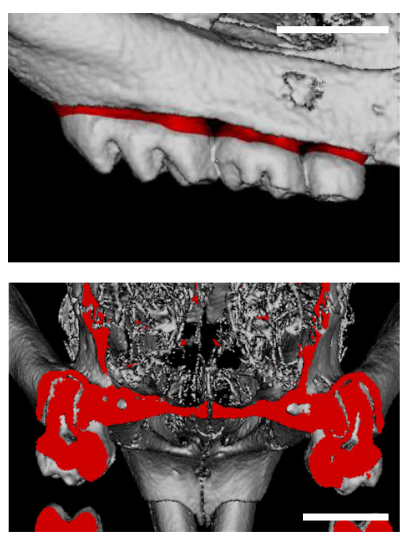

d

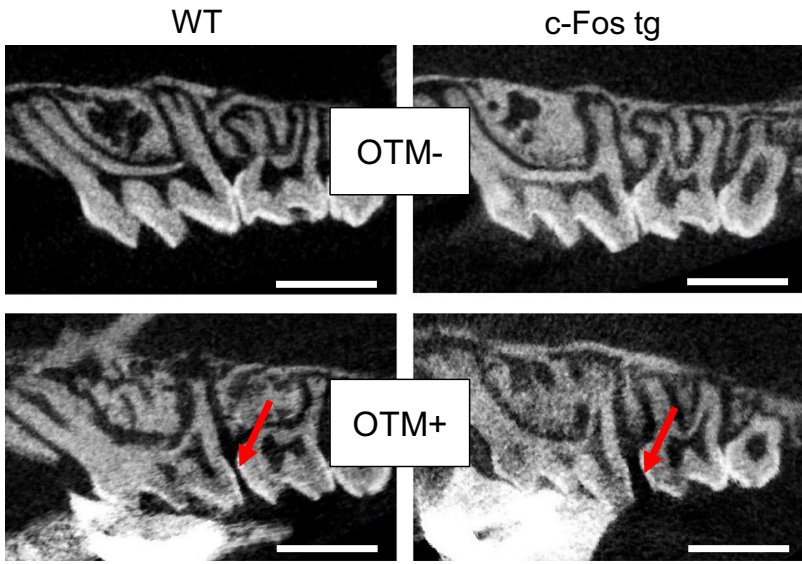

b
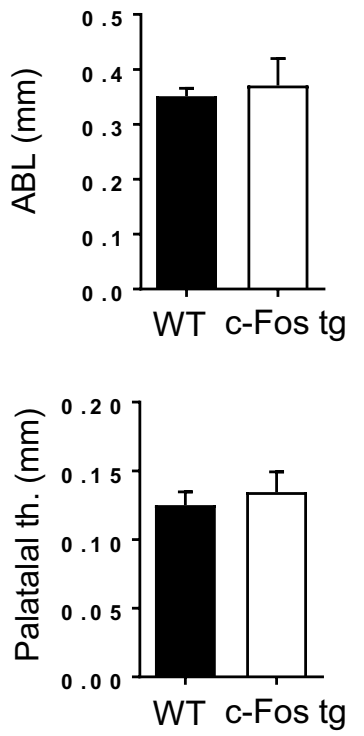

e

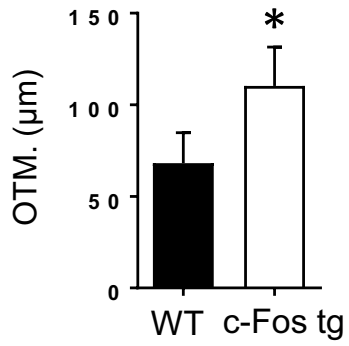

suggested synthesizing activity. Bone-resorbing osteoclasts were also evident on the pressure side in both $c$-Fos tg and control mice (Fig. 3f, lower panels). We further analysed the number and distribution of these osteoclasts by tartrateresistant acid phosphatase (TRAP) staining (Fig. 4). Without OTM, we observed more TRAP-positive cells in the PDL of c-Fos tg as compared with WT (Fig. 4b). These TRAPpositive cells were distributed around the whole root. In contrast, OTM clearly changed the number and distribution of TRAP-positive cells both in c-Fos tg and WT mice as these cells were mainly found in areas of the PDL that were subjected to pressure (Fig. $4 \mathrm{c}$ and $\mathrm{d}$ ). We finally quantified the number of TRAP-positive cells in the PDL of $c-F O S$ tg and WT mice with and without OTM (Fig. 4e). We observed that without OTM, c-Fos tg mice exhibited 40\% more TRAPpositive cells in the PDL as compared with WT. OTM led to a significant increase of TRAP-positive cells in both $c$-Fos tg and WT mice. However, this mechanically induced increase was less pronounced in the teeth of $c-F o s$ tg mice and the number of TRAP-positive cells was therefore significantly lower after OTM in $c$-Fos tg mice as compared with that of WT mice. Taken together, our histological analysis suggests that the acceleration of tooth movement in $c$-Fos tg mice is not mediated by differences in mechanotransduction, but due to a basal increase of bone resorption.

We finally determined whether this increase of bone resorption is also associated with more root resorption in c-Fos tg mice. We again focused here on areas in the PDL that were subjected to orthodontic pressure (Fig. 5a). In comparison with non-stimulated teeth (OTM-), we could clearly detect root resorption in both $c-F o s$ tg mice and WT mice (Fig. $5 \mathrm{~b}$ and c). Interestingly, the shape of these resorptions differed between the two root surfaces. Whereas long, extensive resorptions were noticed at the distal root (Fig. 5c, upper panels), isolated drop-like resorption pits were noticed at the mesial root (Fig. 5c, lower panels). Quantification of root resorptions revealed no significant differences between $c$ Fos tg mice and WT mice (Fig. 5d-f). Root resorptions are usually associated with mechanically induced tissue necrosis. These cell-free, necrotic areas have a glass-like appearance on histological sections and are therefore termed areas of hyalinization [28]. Interestingly, these hyalinizations were evident to a different degree in all WT mice, but in none of the $c$-Fos tg mice (Fig. 5c and g). Taken together, our analysis suggests that the acceleration of OTM in $c-F o s$ tg is not associated with more root resorption. This may be explained 
a

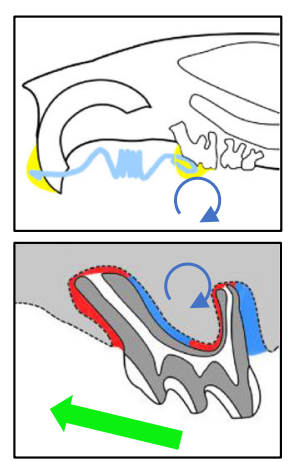

d

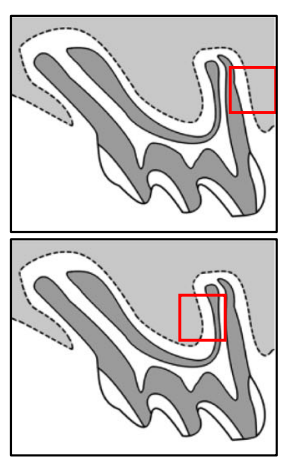

b

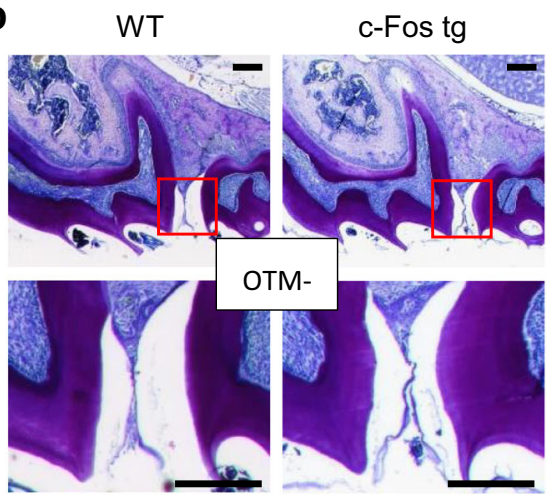

e

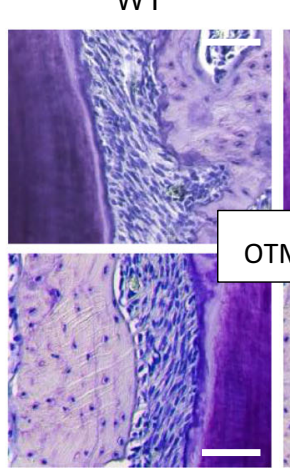
c-Fos tg

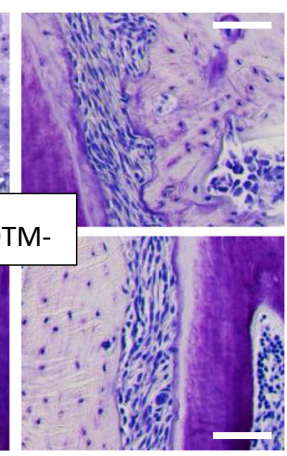

C

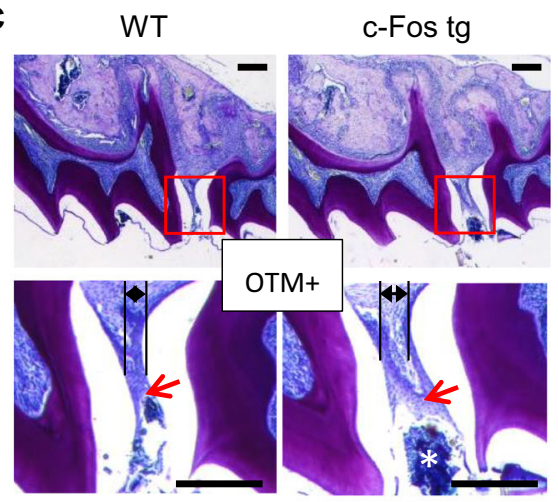

f
WT

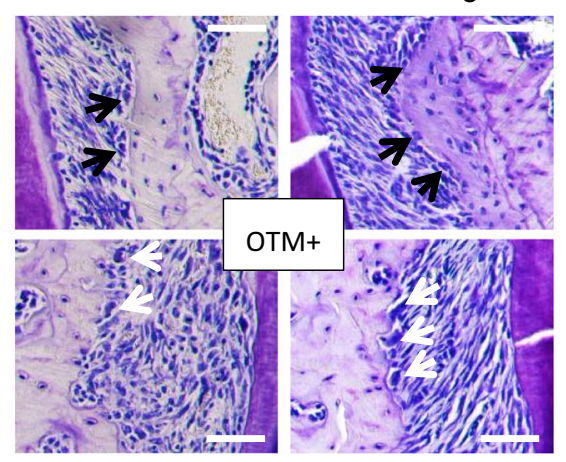

Fig. 3 Histological analysis of OTM in $c$-Fos tg and WT mice. a Schematic drawing of a murine skull (upper panel) and a maxillary first molar (lower panel) illustrating the forces (green arrow) and moments (blue circle) induced by OTM. The direction of the force is mesial and intrusive, which creates different areas of compression (red) and tension (blue). b, c Toluidine-blue stained histological sections of teeth without OTM (b) and with OTM (c) of 12-week-old WT and c-Fos tg mice. Lower panels show magnification of the regions outlined by the red boxes. The intercoronal gaps induced by OTM (black lines) offer a

by the fact that OTM causes less or even no hyalinization in c-Fos tg mice.

\section{Discussion}

This study shows that $c$-Fos plays an important role in the genetic control of tooth movement in vivo. We demonstrate that overexpression of $c$-Fos accelerates OTM in mice without producing more side effects such as root resorption. Since $c$ Fos overexpression did not affect alveolar bone morphology, we believe that the acceleration of OTM in $c$-Fos $t g$ mice can be best explained by a basal increase in bone resorption and the absence of sterile necrosis (i.e. hyalinization).

In fact, the appearance of hyalinization is considered to be an important process in OTM [29]. Based on histological studies, it is assumed that hyalinization is caused by a local disturbance of blood flow in compressed PDL areas. As osteoclast differentiation is impeded in these areas, hyalinization can therefore slow down OTM. This is clearly in line with our retention for debris (white asterisk) resulting in a mild gingivitis (red arrows). Scale bars $=250 \mu \mathrm{m}$. $\mathbf{d}$ Schematic drawing of the maxillary first molar. The red boxes indicate areas of OTM-induced tension (upper panel) and pressure (lower panel) around the distal root. e, f Toluidineblue stained histological sections of the regions indicated in $\mathbf{d}$ of teeth without OTM (e) and with OTM (f) of the same mice. Osteoblasts (black arrow) and osteoclasts (white arrows) are clearly evident after OTM. Scale bars $=50 \mu \mathrm{m}$

findings in c-Fos tg mice, where we observed $62 \%$ faster OTM and no histological evidence of hyalinization. It remains to be established whether $c$-Fos overexpression inhibits the development of hyalinization, or whether it leads to an earlier removal of hyalinization. In this regard, it is also important to mention that $c$-Fos induces angiogenesis [18]. But regardless of this question, we believe that the absence of hyalinization is one major reason for accelerated OTM in $c$-Fos $t g$ mice. This conclusion is further supported by experimental studies showing that surgical interventions to accelerate OTM result in less hyalinization and faster removal of hyalinized tissue [30, 31].

Another reason for faster OTM in c-Fos tg mice could be a basal increase in bone resorption. In fact, our histological analysis of the teeth that were not subjected to tooth movement revealed $40 \%$ more TRAP-positive cells in the PDL of $c$ Fos tg mice as compared with controls. Interestingly, the increase of osteoclastogenesis following OTM was less pronounced in $c-F o s$ tg mice as compared with that in controls. One explanation for this could be that $c-F o s$ transgenic cells are less sensitive to mechanical strain. Another possible 


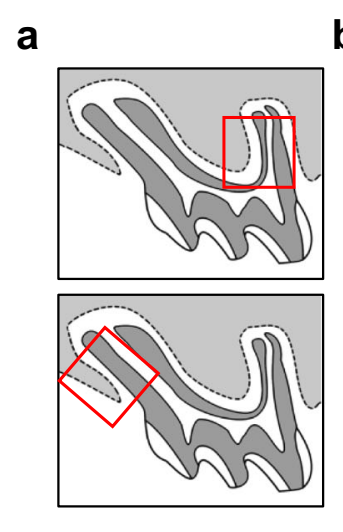

d

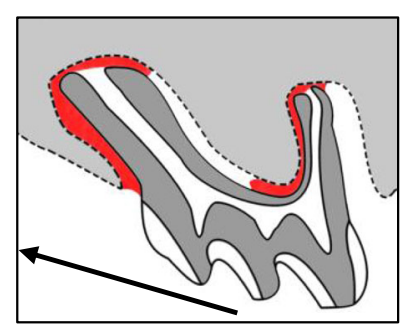

b WT

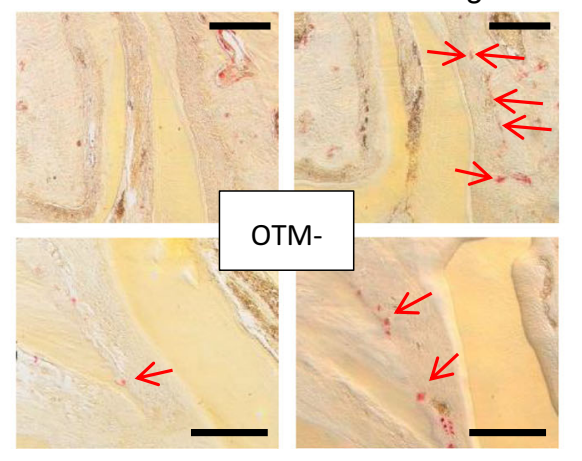

C

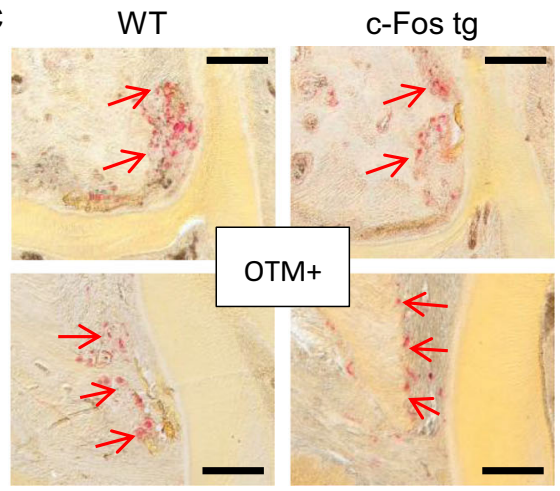

e

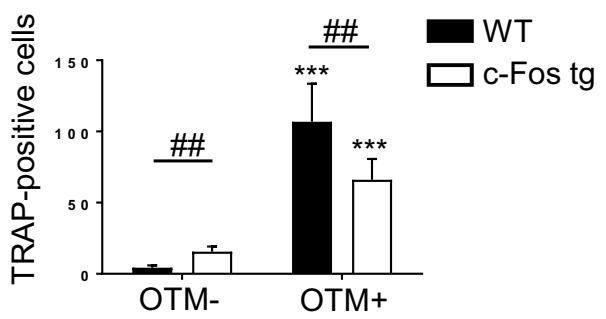

mice. Red arrows indicate TRAP-positive cells. Scale bars $=200 \mu \mathrm{m}$. d Schematic drawing of the maxillary first molar. TRAP-positive cells were quantified in the area indicated in red. e Quantification of TRAP-positive cells. $n=4$. ${ }^{* * *} P<0.001$, versus control. $\# \# P<0.01$, versus wild-type mesial (lower panel) root. b, c TRAP-stained decalcified sections of teeth without OTM (b) and with OTM (c) of 12-week-old WT and c-Fos tg

explanation is that $c$-Fos can inhibit itself by negative feedback via INF- $\beta$ [32]. It remains to be established whether this mechanism also takes place in PDL cells during OTM. In this regard, it is also important to mention that the load-induced expression of $c-F o s$, as an early response gene, is both rapid and short lived. Given the time frame of our experiments, it is clear that our results cannot directly be correlated to previous in vitro studies analysing the short-term response of $c$-Fos to mechanical stress [17-23]. Future studies should address this question by performing a short-term OTM in $c-F o s$ tg mice following an immunohistological analysis of target genes.

One important histological observation was the occurrence of lateral root resorptions, which is a common side effect of orthodontic therapy [33]. Quantification of this root resorption in $c$-Fos tg and control mice using histomorphometry revealed no significant differences. This is an important finding as it demonstrates that the acceleration of OTM in $c-F o s$ tg mice does not cause more root resorption. It was interesting to observe that the shape of lateral root resorption differed with regard to its location. Whereas root resorptions extended along almost the entire surface of the distal root, only isolated drop-like resorption pits were observed at the mesial root. A possible explanation for this could be that the tissue pressure induced by OTM differs between the mesial and the distal root. In fact, the distal surface of the distal root has a concave shape and the surrounding bone follows the root curvature.
Therefore, OTM presumably creates a homogenous tissue pressure along the entire root surface. In contrast, the mesial surface of the mesial root has a convex shape and the surrounding bone does not follow entirely the root curvature, which means that the PDL is thinner at the root cervix as compared with the apical part of the root. Therefore, OTM presumably creates exceeding tissue pressure only in this cervical part. This is line with our findings as we observed only in this area root resorption and hyalinization. We believe that the correlation between root resorption and PDL morphology warrants further studies.

All these histological observations clearly demonstrate the relevance of genetically modified mice for orthodontic research. Our experimental protocol for the OTM model was based on previous studies by Taddei et al., Braga et al. and Andrade et al. [8, 9, 34-36]. According to this protocol, we bonded the Nitinol spring to the teeth using a light-curing composite. In contrast, other authors fixed the Nitinol spring with a wire ligature around the first molar $[6,7,37,38]$. Although this might be easier to perform, we believe that wire ligatures have several disadvantages. Firstly, wire ligatures produce metal artefacts in the micro-CT scanning and these artefacts are in the interproximal area of interest. Although metal artefacts were also evident in our micro-CT scans, these were on the occlusal surface of the first molar and did not affect our quantification of the micro-CT scans. Secondly, 
a

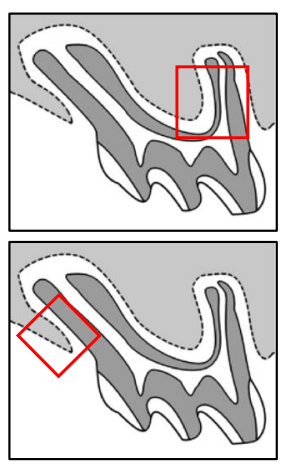

d

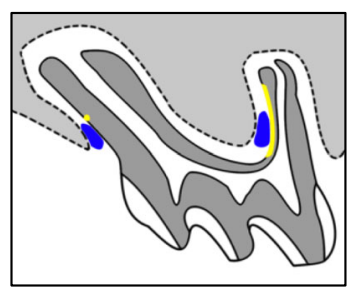

b

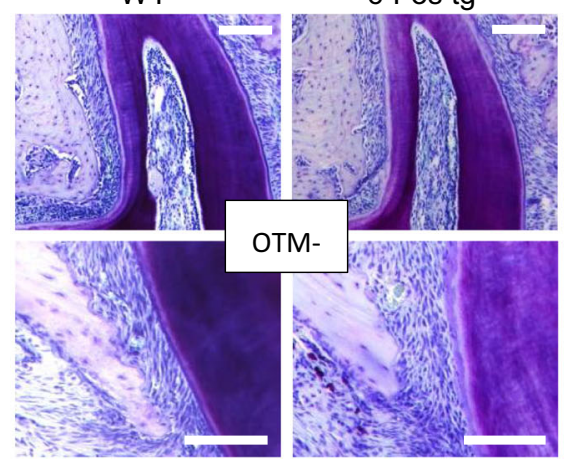

C

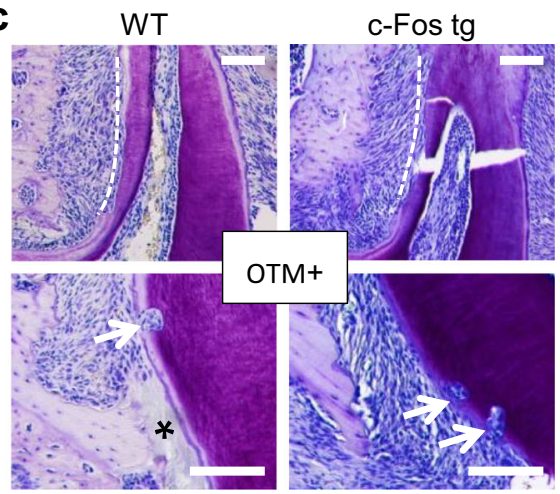

e

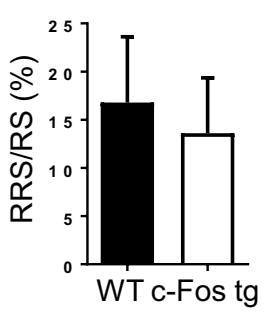

$f$

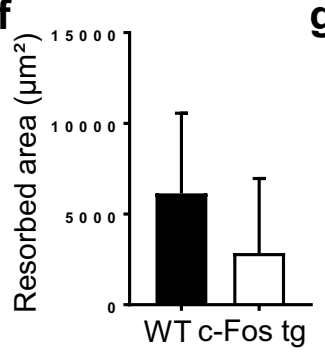

g

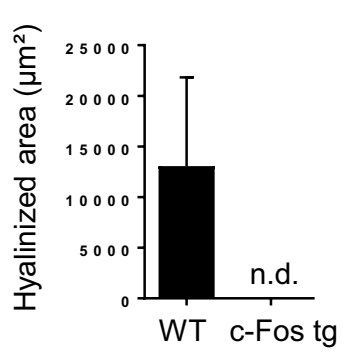

Fig. 5 Histological analysis of OTM-induced root resorption and hyalinization in $c$-Fos tg and WT mice. a Schematic drawing of the maxillary first molar. The red boxes indicate areas of OTM-induced pressure at the distal (upper panel) and mesial (lower panel) root. b, c Toluidine-blue stained histological sections of teeth without OTM (b) and with OTM (c) of 12-week-old WT and c-Fos tg mice. Scale bars = $100 \mu \mathrm{m}$. Whereas lateral resorption at the distal root extends along almost

wire ligatures can create debris niche and cause periodontal destruction during OTM. Indeed, ligatures around molars are an established model to induce periodontitis in mice [39]. Unfortunately, this ligature-induced periodontitis can also affect OTM [40]. We also noted gingival inflammation, but this inflammation was limited to the intercoronal gap and caused by the OTM and not by the appliance itself. Finally, we believe that bonding of the wire is less invasive than wire ligatures, which can cause mucous tissue injury due to the interdental threading of the wire. Accordingly, our daily weight control showed that the animals were not exposed to any serious stress. In this regard, it is also important to mention that our split-mouth designs using the contralateral side as internal controls significantly reduced the number of experimental animals as compared with other studies [41, 42].

Nevertheless, our study has certain limitations. One limitation is that our study only addresses how overexpression of $c$ Fos affects OTM. Of course, it would also be interesting to analyse whether decreased expression of $c-F o s$ has an effect on OTM. However, the deletion of $c-F o s$ in mice blocks osteoclastic differentiation and the dental phenotype of c-Fosdeficient mice is therefore characterized by a lack of tooth eruption and a lack of root formation $[12,43]$. It is therefore not possible to perform OTM in these mice. Another limitation of our study is that $c$-Fos $\operatorname{tg}$ mice are characterized by the the entire root surface (white dotted line), lateral resorption at the mesial root has a drop-like appearance (white arrow). Areas of hyalinization (black asterisk) were only evident in WT mice. Scale bars $=100 \mu \mathrm{m}$. d Schematic drawing of the maxillary first molar. E-G Quantification of the resorbed root surface per root surface (RRS/RS), resorbed area and hyalinized area in the PDL of 12-week-old WT and c-Fos tg mice. $n=4$

development of chondrogenic bone tumours, which may have a general effect on bone metabolism [15]. These benign tumours initially occur mainly in the tubular bones of the extremities, but with increasing age, they can also be found in the vertebral bodies and ribs [25, 27, 44-48]. It was therefore important to analyse the alveolar bone of $c$-Fos tg mice without OTM. We did not observe any of these tumours in the jaws of $c$-Fos $t g$ mice. This finding can be explained by the fact that jaws are formed through intramembranous ossification. Furthermore, our quantification of alveolar bone loss and palatal thickness using $\mu-\mathrm{CT}$ imaging revealed no significant differences between WT and c-Fos tg mice. This is an important finding as it demonstrated that differences in alveolar bone structure cannot explain the faster OTM in c-Fos tg mice. Finally, we would like to mention that the number of animals used for this study is comparatively low and it is possible that more significant differences would have been found with a larger sample size.

\section{Conclusion}

Our study demonstrates that $c$-Fos overexpression in mice accelerates tooth movement due to a basal increase in bone resorption and the inhibition of mechanically induced tissue 
necrosis. Importantly, the faster OTM in c-Fos $t g$ mice was not associated with more root resorption. We believe that this finding is also relevant with regard to surgically accelerated OTM as it demonstrates that these procedures must not result in more adverse effects such as root resorption. Future studies should use genetically modified mice to further analyse the genetic regulation of OTM.

Acknowledgements We thank Elke Leicht, Mona Neven, Lana Rosenthal and Olga Winter for technical assistance. The authors also thank Kimberly Oppenheim for critically reading the manuscript. This article is in memory of our dear colleague and friend Dr. Jean-Pierre David, who passed away during the revision of this manuscript.

Funding information Open Access funding provided by Projekt DEAL.

\section{Compliance with ethical standards}

Conflict of interest The authors declare that they have no conflict of interest.

Research involving animals Animal procedures were performed in accordance with the commission for animal welfare (Behörde für Gesundheit und Verbraucherschutz der Hansestadt Hamburg, Nr. 121/16).

Open Access This article is licensed under a Creative Commons Attribution 4.0 International License, which permits use, sharing, adaptation, distribution and reproduction in any medium or format, as long as you give appropriate credit to the original author(s) and the source, provide a link to the Creative Commons licence, and indicate if changes were made. The images or other third party material in this article are included in the article's Creative Commons licence, unless indicated otherwise in a credit line to the material. If material is not included in the article's Creative Commons licence and your intended use is not permitted by statutory regulation or exceeds the permitted use, you will need to obtain permission directly from the copyright holder. To view a copy of this licence, visit http://creativecommons.org/licenses/by/4.0/.

\section{References}

1. Paul GR, Malhotra A, Müller R (2018) Mechanical stimuli in the local in vivo environment in bone: computational approaches linking organ-scale loads to cellular signals. Curr Osteoporos Rep 16:395-403

2. Pilon JJGM, Kuijpers-Jagtman AM, Maltha JC (1996) Magnitude of orthodontic forces and rate of bodily tooth movement. An experimental study. Am J Orthod Dentofac Orthop 110:16-23. https:// doi.org/10.1016/S0889-5406(96)70082-3

3. Giannopoulou C, Dudic A, Pandis N, Kiliaridis S (2016) Slow and fast orthodontic tooth movement: an experimental study on humans. Eur J Orthod 38:404-408. https://doi.org/10.1093/ejo/ cjv070

4. Küchler EC, Schröder A, Corso P, Scariot R, Spanier G, Proff P, Kirschneck C (2019) Genetic polymorphisms influence gene expression of human periodontal ligament fibroblasts in the early phases of orthodontic tooth movement. Odontology 108:1-10. https://doi.org/10.1007/s10266-019-00475-x

5. Al-Qawasmi RA, Hartsfield JK, Everett ET, Flury L, Liu L, Foroud TM, Macri JV, Roberts WE (2003) Genetic predisposition to external apical root resorption. Am J Orthod Dentofac Orthop 123:242-252. https://doi.org/10.1067/mod.2003.42

6. Al-Qawasmi RA, Hartsfield JK, Everett ET, Weaver MR, Foroud TM, Faust DM, Roberts WE (2006) Root resorption associated with orthodontic force in inbred mice: genetic contributions. Eur J Orthod 28:13-19. https://doi.org/10.1093/ejo/cji090

7. Al-Qawasmi RA, Hartsfield JK, Everett ET, Weaver MR, Foroud TM, Roberts WE (2004) Root resorption associated with orthodontic force in IL-1B knockout mouse. J Musculoskelet Neuronal Interact 4:383-385

8. Braga SMG, De Albuquerque Taddei SR, Andrade I, QueirozJunior CM, Garlet GP, Repeke CE, Teixeira MM, Da Silva TA (2011) Effect of diabetes on orthodontic tooth movement in a mouse model. Eur J Oral Sci 119:7-14. https://doi.org/10.1111/j. 1600-0722.2010.00793.x

9. Taddei SR d A, Moura AP, Andrade I, Garlet GP, Garlet TP, Teixeira MM, da Silva TA (2012) Experimental model of tooth movement in mice: a standardized protocol for studying bone remodeling under compression and tensile strains. J Biomech 45: 2729-2735. https://doi.org/10.1016/j.jbiomech.2012.09.006

10. Wolf M, Ao M, Chavez MB, Kolli TN, Thumbigere-Math V, Becker K, Chu EY, Jäger A, Somerman MJ, Foster BL (2018) Reduced orthodontic tooth movement in Enpp1 mutant mice with hypercementosis. J Dent Res 97:937-945. https://doi.org/10.1177/ 0022034518759295

11. Yang CY, Jeon HH, Alshabab A, Lee YJ, Chung CH, Graves DT (2018) RANKL deletion in periodontal ligament and bone lining cells blocks orthodontic tooth movement. Int J Oral Sci 10:1-9. https://doi.org/10.1038/s41368-017-0004-8

12. Boyce BF, Yamashita T, Yao Z, Zhang Q, Li F, Xing L (2005) Roles for NF-kB and c-Fos in osteoclasts. J Bone Miner Metab 23: $11-15$

13. Wang Z-Q, Ovitt C, Grigoriadis AE, Möhle-Steinlein U, Rüther U, Wagner EF (1992) Bone and haematopoietic defects in mice lacking c-fos. Nature 360:741-745. https://doi.org/10.1038/360741a0

14. Grigoriadis AE, Wang ZQ, Cecchini MG, Hofstetter W, Felix R, Fleisch HA, Wagner EF (1994) c-Fos: a key regulator of osteoclastmacrophage lineage determination and bone remodeling. Science 266:443-448. https://doi.org/10.1126/SCIENCE.7939685

15. Grigoriadis AE, Schellander K, Wang ZQ, Wagner EF (1993) Osteoblasts are target cells for transformation in c-fos transgenic mice. J Cell Biol 122:685-701

16. Peake MA, Cooling LM, Magnay JL, Thomas PBM, El Haj AJ (2000) Selected contribution: regulatory pathways involved in mechanical induction of c-fos gene expression in bone cells. J Appl Physiol 89:2498-2507. https://doi.org/10.1152/jappl.2000.89.6. 2498

17. Kawata A, Mikuni-Takagaki Y (1998) Mechanotransduction in stretched osteocytes - temporal expression of immediate early and other genes. Biochem Biophys Res Commun 246:404-408. https:// doi.org/10.1006/bbrc.1998.8632

18. Mason DJ, Hillam RA, Skerry TM (2009) Constitutive in vivo mRNA expression by osteocytes of $\beta$-actin, osteocalcin, connexin-43, IGF-I, c-fos and c-jun, but not TNF- $\alpha$ nor tartrateresistant acid phosphatase. J Bone Miner Res 11:350-357. https:// doi.org/10.1002/jbmr.5650110308

19. Moalli MR, Caldwell NJ, Patil PV, Goldstein SA (2000) An in vivo model for investigations of mechanical signal transduction in trabecular bone. J Bone Miner Res 15:1346-1353. https://doi.org/10. 1359/jbmr.2000.15.7.1346

20. Li J, Chen G, Zheng L, Luo S, Zhao Z (2007) Osteoblast cytoskeletal modulation in response to compressive stress at physiological levels. Mol Cell Biochem 304:45-52. https://doi.org/10.1007/ s11010-007-9484-8

21. Glantschnig H, Varga F, Rumpler M, Klaushofer K (1996) Prostacyclin (PGI2): a potential mediator of c-fos expression 
induced by hydrostatic pressure in osteoblastic cells. Eur J Clin Invest 26:544-548

22. Pavalko FM, Chen NX, Turner CH, Burr DB, Atkinson S, Hsieh YF, Qiu J, Duncan RL (1998) Fluid shear-induced mechanical signaling in MC3T3-E1 osteoblasts requires cytoskeleton-integrin interactions. Am J Physiol 275:C1591-C1601

23. Sen S, Diercke K, Zingler S, Lux CJ, Erber R (2015) Compression induces Ephrin-A2 in PDL fibroblasts via c-fos. J Dent Res 94:464 472. https://doi.org/10.1177/0022034514567197

24. Hanahan D, Wagner EF, Palmiter RD (2007) The origins of oncomice: a history of the first transgenic mice genetically engineered to develop cancer. Genes Dev 21:2258-2270. https:// doi.org/10.1101/GAD.1583307

25. Rüther U, Garber C, Komitowski D, Müller R, Wagner EF (1987) Deregulated c-fos expression interferes with normal bone development in transgenic mice. Nature 325:412-416. https://doi.org/10. $1038 / 325412 \mathrm{a} 0$

26. Morello D, Moore G, Salmon AM, Yaniv M, Babinet C (1986) Studies on the expression of an H-2K/human growth hormone fusion gene in giant transgenic mice. EMBO J 5:1877-1883. https:// doi.org/10.1002/j.1460-2075.1986.tb04439.x

27. David J-P, Mehic D, Bakiri L, Schilling AF, Mandic V, Priemel M, Idarraga MH, Reschke MO, Hoffmann O, Amling M, Wagner EF (2005) Essential role of RSK2 in c-Fos-dependent osteosarcoma development. J Clin Invest 115:664-672. https://doi.org/10.1172/ JCI22877

28. Reitan K (1967) Clinical and histologic observations on tooth movement during and after orthodontic treatment. Am J Orthod 53:721-745

29. Von Böhl M, Kuijpers-Jagtman AM (2009) Hyalinization during orthodontic tooth movement: a systematic review on tissue reactions. Eur J Orthod 31:30-36. https://doi.org/10.1093/ejo/cjn080

30. Kim SJ, Park YG, Kang SG (2008) Effects of corticision on paradental remodeling in orthodontic tooth movement. Angle Orthod 79:284-291. https://doi.org/10.2319/020308-60.1

31. Cano J, Campo J, Bonilla E, Colmenero C (2012) Corticotomyassisted orthodontics. J Clin Exp Dent 4:e54-e59. https://doi.org/ $10.4317 /$ jced.50642

32. Takayanagi H, Kim S, Matsuo K, Suzuki H, Suzuki T, Sato K, Yokochi T, Oda H, Nakamura K, Ida N, Wagner EF, Taniguchi T (2002) RANKL maintains bone homeostasis through c-fosdependent induction of interferon- $\beta$. Nature 416:744-749. https:// doi.org/10.1038/416744a

33. Killiany DM (1999) Root resorption caused by orthodontic treatment: an evidence-based review of literature. Semin Orthod 5:128133

34. Andrade I, Silva TA, Silva GAB, Teixeira AL, Teixeira MM (2007) The role of tumor necrosis factor receptor type 1 in orthodontic tooth movement. J Dent Res 86:1089-1094. https://doi.org/10. $1177 / 154405910708601113$

35. Andrade I, Taddei SRA, Garlet GP, Garlet TP, Teixeira AL, Silva TA, Teixeira MM (2009) CCR5 down-regulates osteoclast function in orthodontic tooth movement. J Dent Res 88:1037-1041. https:// doi.org/10.1177/0022034509346230

36. Taddei SR d A, Queiroz-Junior CM, Moura AP, Andrade I, Garlet GP, Proudfoot AEI, Teixeira MM, da Silva TA (2013) The effect of
CCL3 and CCR1 in bone remodeling induced by mechanical loading during orthodontic tooth movement in mice. Bone 52:259-267. https://doi.org/10.1016/j.bone.2012.09.036

37. Yoshimatsu M, Shibata Y, Kitaura H, Chang X, Moriishi T, Hashimoto F, Yoshida N, Yamaguchi A (2005) Experimental model of tooth movement by orthodontic force in mice and its application to tumor necrosis factor receptor-deficient mice. J Bone Miner Metab 24:20-27. https://doi.org/10.1007/s00774-005-0641-4

38. Matsumoto T, Iimura T, Ogura K, Moriyama K, Yamaguchi A (2013) The role of osteocytes in bone resorption during orthodontic tooth movement. J Dent Res 92:340-345. https://doi.org/10.1177/ 0022034513476037

39. Abe T, Hajishengallis G (2013) Optimization of the ligatureinduced periodontitis model in mice. J Immunol Methods 394: 49-54. https://doi.org/10.1016/j.jim.2013.05.002

40. Okamoto A, Ohnishi T, Bandow K, Kakimoto K, Chiba N, Maeda A, Fukunaga T, Miyawaki S, Matsuguchi T (2009) Reduction of orthodontic tooth movement by experimentally induced periodontal inflammation in mice. Eur J Oral Sci 117:238-247. https://doi.org/ 10.1111/j.1600-0722.2009.00625.x

41. Saitoh S, Takahashi I, Mizoguchi I, Sasano Y, Kagayama M, Mitani H (2000) Compressive force promotes chondrogenic differentiation and hypertrophy in midpalatal suture cartilage in growing rats. Anat Rec 260:392-401. https://doi.org/10.1002/10970185(20001201)

42. Madan MS, Liu ZJ, Gu GM, King GJ (2007) Effects of human relaxin on orthodontic tooth movement and periodontal ligaments in rats. Am J Orthod Dentofac Orthop 131:8.e1-8.e10. https://doi. org/10.1016/J.AJODO.2006.06.014

43. Alfaqeeh S, Oralova V, Foxworthy M, Matalova E, Grigoriadis AE, Tucker AS (2015) Root and eruption defects in $c$-Fos mice are driven by loss of osteoclasts. J Dent Res 94:1724-1731. https:// doi.org/10.1177/0022034515608828

44. Rüther U, Komitowski D, Schubert FR, Wagner EF (1989) c-fos expression induces bone tumors in transgenic mice. Oncogene 4 : 861-865

45. Rauscher FJ, Voulalas PJ, Franza BR, Curran T (1988) Fos and Jun bind cooperatively to the AP-1 site: reconstitution in vitro. Genes Dev 2:1687-1699. https://doi.org/10.1101/gad.2.12b.1687

46. Wang ZQ, Liang J, Schellander K, Wagner EF, Grigoriadis AE (1995) c-fos-induced osteosarcoma formation in transgenic mice: cooperativity with c-jun and the role of endogenous c-fos. Cancer Res 55:6244-6251

47. Wagner EF, Matsuo K (2003) Signalling in osteoclasts and the role of Fos/AP1 proteins. Annals of the Rheumatic Diseases. BMJ Publishing Group Ltd, In, pp 83-85

48. Leaner VD, Chick JF, Donninger H, Linniola I, Mendoza A, Khanna C, Birrer MJ (2009) Inhibition of AP-1 transcriptional activity blocks the migration, invasion, and experimental metastasis of murine osteosarcoma. Am J Pathol 174:265-275. https://doi.org/ 10.2353/ajpath.2009.071006

Publisher's note Springer Nature remains neutral with regard to jurisdictional claims in published maps and institutional affiliations. 